\title{
Field Synergy Analysis of Mass Transfer Characteristics of Vibrating Hollow Fiber Membrane Modules
}

\author{
ZHANG XiaoGuang ${ }^{a}$, GAI PengXing ${ }^{b}$, WANG ShiGang ${ }^{c}$ and LIU Li $^{d}$ \\ College of Electromechanical Engineering, Qingdao University of Science \& Technology, Qingdao \\ 266061, Shandong Province, P.R.China \\ axgzhang79@gmail.com, bgaipengxing@163.com, 'taiwang-wsg@163.com, d214934965@qq.com
}

Keywords: hollow fiber membrane modules; mass transfer; vibration condition; numerical simulation; field synergy principle

Abstract. In this paper, the field synergy principle is used to simulate the mass transfer performance of hollow fiber membrane modules under vibration conditions. The mass transfer characteristics of hollow fiber membrane modules are investigated by changing the vibration frequency, amplitude and fluid velocity. The results prove that: the average field synergy number under vibration conditions is much higher than that under the non-vibration condition. The average field synergy number increases with the frequency and amplitude. But the increase of vibration frequency and amplitude does not linearly improve the mass transfer performance of the hollow fiber membrane modules. Compared with the increase of amplitude, the enhancement effect of increasing frequency on mass transfer is more obvious. With the increase of the vibration energy consumption, the mass transfer efficiency of the hollow fiber modules increases. With the increase of flow velocity, the average field synergy number shows a decreasing trend, meaning that the degree of synergy between the velocity field and the concentration field decreases. While the Reynolds number will increase with the flow velocity, which will enhance the mass transfer performance of the hollow fiber membrane modules.

\section{Introduction}

The field synergy principle was first proposed by Guo, et al[1], the principle reveals that the intensity of the equivalent heat source in the convective heat transfer process depends not only on the velocity field and temperature gradient field but also on their synergy. The intensity of the equivalent heat source is dependent on the included angle between the velocity field and temperature gradient field. The smaller the included angle (less than 90 degrees) is, the better synergy of velocity and temperature gradient fields is. Better synergy will lead to a larger value of the dimensionless equivalent heat source, and the convection heat transfer performance is enhanced, which can be called the field of collaborative [2-5].

In recent years, the field synergy principle has been applied mainly in the convective heat transfer fields[6-12]. Because the mass transfer process and heat transfer process have many similar laws, the field synergy principle of convective heat transfer process can be extended to the convective mass transfer process. $\mathrm{Wu}[13]$ derived the field synergy equation of convective heat and mass transfer. Meng[14] quantitatively evaluated the effects of Reynolds number and mixed member structure on the synergy of velocity field and pressure field in a static mixer by means of the integral mean angle. In this paper, based on the field synergy principle, the heat transfer enhancement is extended to the mass transfer process. The mass transfer performance of hollow fiber membrane modules under different vibration conditions were analyzed by means of the numerical simulation.

\section{Model}

Physical Model. In this paper, the physical model was selected as the mass transfer process of which the dissolved oxygen was removed from water in the hollow fiber membrane modules. As shown in Fig.1, oxygen-containing water flowed along the outside of the hollow fiber tubes, and nitrogen flowed along the inside of hollow fiber tubes counter-currently. It was assumed that the hollow fiber tubes were arranged regularly, the radius of the membrane modules was $10 \mathrm{~mm}$, the radius of the fiber 
tubes was $1 \mathrm{~mm}$, the length of the membrane modules and the fiber tubes was $500 \mathrm{~mm}$. In order to observe the effect of vibration conditions on the mass transfer performance of membrane modules, the regular arrangement of hollow fiber tubes in the lower packing density (5\%) was adopted.
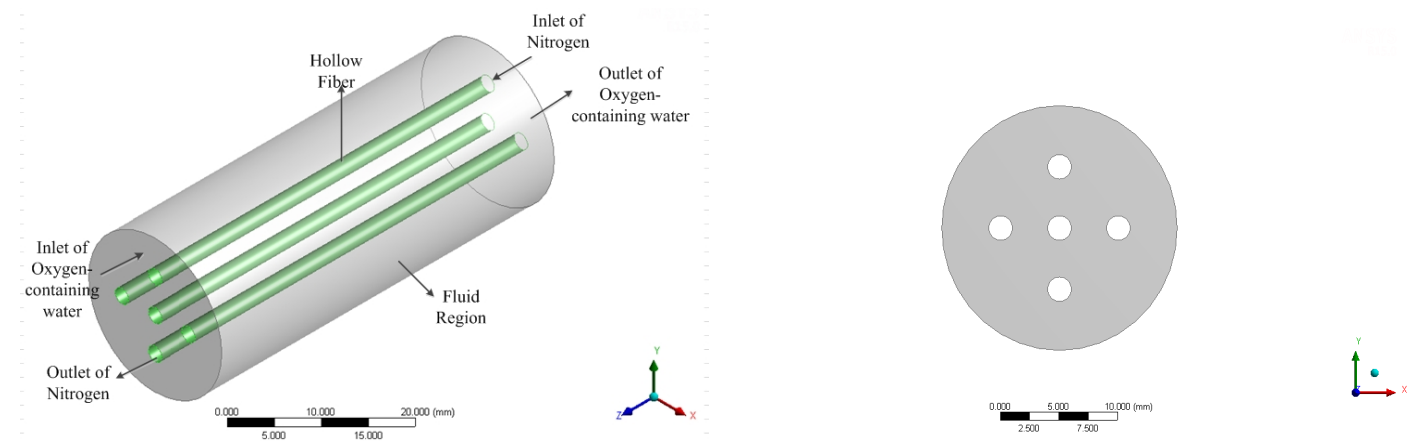

Fig.1 Typical hollow fiber modules and its cross section

Mathematical Model. The model was developed with the following assumptions: (1)The oxygen-containing water was the incompressible Newton fluid; (2) The gravity and buoyancy were ignored; (3) The system was operated isothermally and in a steady state condition; (4) Physical properties of the fluid and the fibers were constant; (5) The vibration of the hollow fiber tubes was a simple harmonic motion, the motion parameters depended on the amplitude and frequency.

Based on the above assumptions, the dimensionless relationship of the laminar convective mass transfer performance in steady-state and non-component source terms is [2]:

$$
S h=\operatorname{Re} S c \int_{\Omega} \bar{U} \cdot \nabla \bar{Y} d \bar{V}
$$

Where, $S h$ is the dimensionless Sherwood number, which reflects the intensity of the convective mass transfer performance; $U$ is the velocity of fluid, $Y$ is the concentration of oxygen, $V$ is the volume of modle.

Eq. (1) indicates that the intensity of convective mass transfer depends not only on the value of $R e$ and $S c$, but also on the integral $\bar{U} \cdot \nabla \bar{Y}$ in the whole mass transfer region. The field synergy number is:

$$
F c=\int_{\Omega} \bar{U} \cdot \nabla \bar{Y} d \bar{V}
$$

It represents the degree of synergy between the velocity field and the concentration field (concentration gradient field).

$$
\bar{U} \cdot \nabla \bar{Y}=|\bar{U}| \cdot|\nabla \cdot \bar{Y}| \cdot \cos \beta
$$

Where, $\beta$ is the included angle between the velocity field and concentration field, which is known as the field synergy angle. It can be seen from Eq.(3), reducing the included angle is an effective way to improve the convective mass transfer performance.

The formula of local field synergy angle is:

$$
\beta_{\phi}=\arccos \left(\frac{u \frac{\partial Y}{\partial x}+v \frac{\partial Y}{\partial y}+w \frac{\partial Y}{\partial z}}{|\bar{U}||\operatorname{grad} Y|}\right)
$$

The average field synergy angle is used to evaluate the degree of synergy between the velocity field and concentration gradient field in the whole region. The formula is: 


$$
\beta=\sum \frac{|\bar{u}|_{i} \cdot|\operatorname{grady}|_{i} \cdot d V_{i}}{\sum|\vec{u}|_{i} \cdot|\operatorname{grady}|_{i} \cdot d V} \cdot \beta_{\phi}
$$

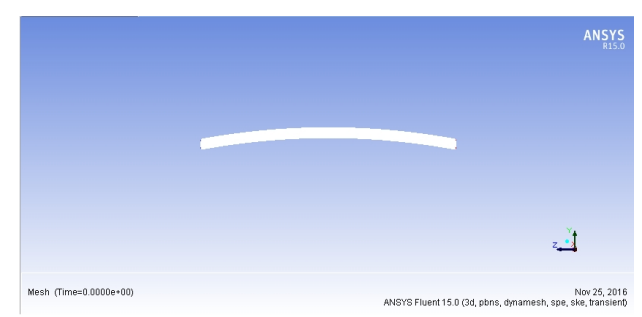

Fig.2 The hollow fiber module vibration

In the Cartesian coordinate system as shown in Fig.2, the hollow fiber tubes undergo a simple harmonic vibration in the $y$ direction [8]:

$$
y(z, t)=A z(0.5-z) \sin (2 \pi f t) / L
$$

Where, $y, z$ are the hollow fiber tube coordinates; $t$ is the time of vibration; $\mathrm{A}$ is the amplitude; $f$ is the vibration frequency; $\mathrm{L}$ is a constant, which is related to the length of the fiber tube, with the number of 0.0625 in this study.

\section{Numerical Simulations}

Numerical simulations were conducted by using computational fluid dynamics software FLUENT, and the governing equations were solved by uncoupled transient implicit scheme. At the entrance, the boundary condition was inlet velocity and the concentration of oxygen was $1 \%$. The outlet condition was a free flow. The concentration of the oxygen around the surface of the hollow fibers was 0 . In addition, SIMPLEC algorithm was adopted into the coupling of pressure and velocity, and the vibration mode of the hollow fiber tubes was defined by UDF. The vibration amplitudes of $2 \mathrm{~mm}$, $4 \mathrm{~mm}, 6 \mathrm{~mm}$ and frequencies of $2.5 \mathrm{~Hz}, 5 \mathrm{~Hz}$ and $10 \mathrm{~Hz}$ were set for this study.

In the middle position of the hollow fiber modules, the velocity vectors at the cross section with the phase of 90 degree are shown in Fig.3. It can be seen that there is a secondary flow in the fluid, and the direction of the arrow is significantly different from that of the original fluid.

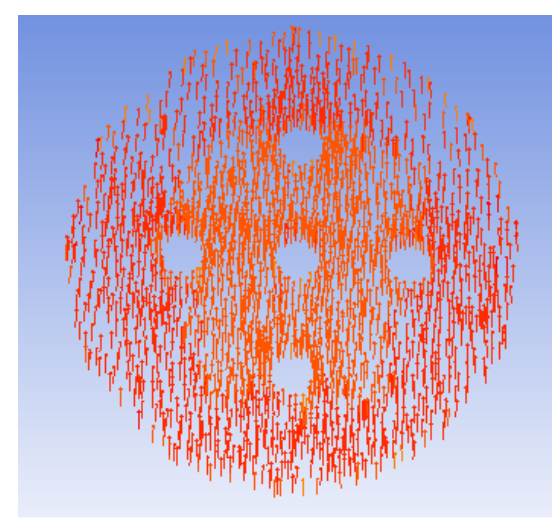

Fig.3 Velocity vectors at the cross section 


\section{Results and Analysis}

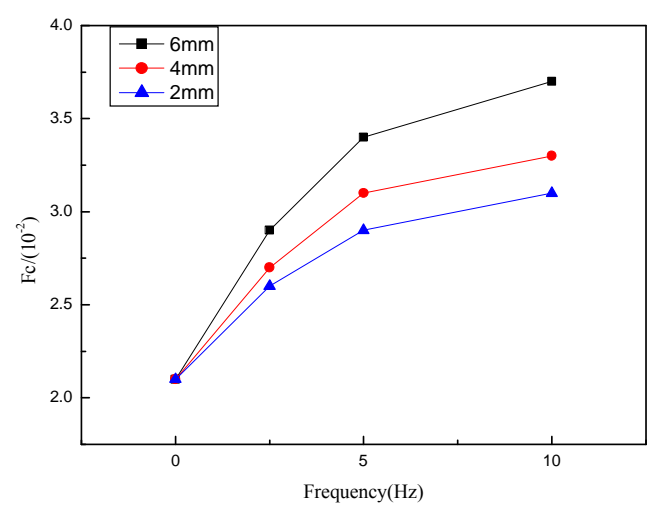

Fig.4 The average field synergy number with vibration frequency and amplitude

Effect of Amplitude and Frequency on the Synergy Degree. The variation of the average field synergy number under different vibration parameters was shown in Fig.4. The average field synergy number under vibration condition is obviously higher than that under the non-vibration condition. With the increase of the vibration frequency and amplitude, the average field synergy number increases.

When the vibration frequency is held constant, with the increase of the amplitude, the climbing speed of field synergy number tends to slow down. Instead, when the amplitude is held constant, the field synergy number goes up with the increase of the frequency. Therefore, the increase of vibration frequency and amplitude does not linearly improve the mass transfer performance of the hollow fiber membrane modules.

Effect of Vibration Energy Consumption on Mass Transfer Performance. For a vibration system with simple harmonic motion, the energy consumption is obtained by the equation of the vibration energy of the harmonic motion [15]:

$$
E=\frac{1}{2} k A^{2}
$$

Where, $k$ is the stiffness coefficient, $k=m \cdot k_{0}{ }^{2}\left(f=\frac{1}{2 \pi} \cdot \frac{k}{m}\right), k_{0}$ is the natural frequency of vibration system (or the nature of vibration system), $m$ is the mass of vibrator.

Eq. (7) indicates that the energy consumption of vibration system is:

$$
E=\frac{1}{2} k A^{2}=2 \pi^{2} f^{2} m A^{2}=2 \pi^{2} m(A \cdot f)^{2}
$$

From Eq.(8), it can be concluded that the vibration energy consumption of hollow fiber membrane modules is related to the amplitude and frequency of vibration system. With the increase of the product values of the amplitude and frequency, the energy consumption of the vibration rises gradually. 


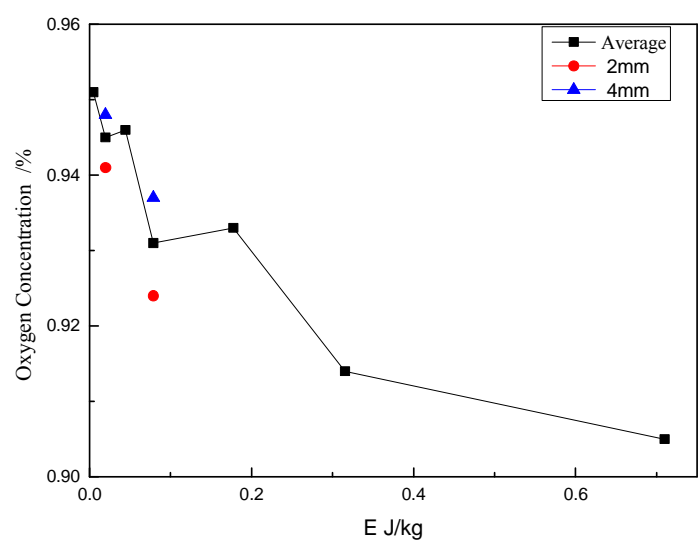

Fig. 5 The outlet oxygen concentration with vibration energy consumption

The variation of the outlet oxygen concentration under different vibration parameters was shown in Fig.5. With the increase of the vibration energy consumption per unit mass, the outlet oxygen concentration shows a tendency of decrease. The reason is that the velocity of the oxygen-containing water along oxygen concentration gradient increases with the vibration energy consumption, which reduces the included angle and enlarges the degree of synergy between the concentration field and velocity field. It improves the effect of the absent dissolved oxygen in the hollow fiber membrane modules.

Comparing both the blue and the red point in Fig.5, it can also be concluded that at the same product values of the amplitude and frequency, the outlet oxygen concentration with the increasing of the amplitude is higher than that with the increasing of the frequency. Therefore, in the range of the parameters which were studied in this paper, the effect of increasing the vibration frequency on the convective mass transfer performance is better than that of increasing the amplitude.

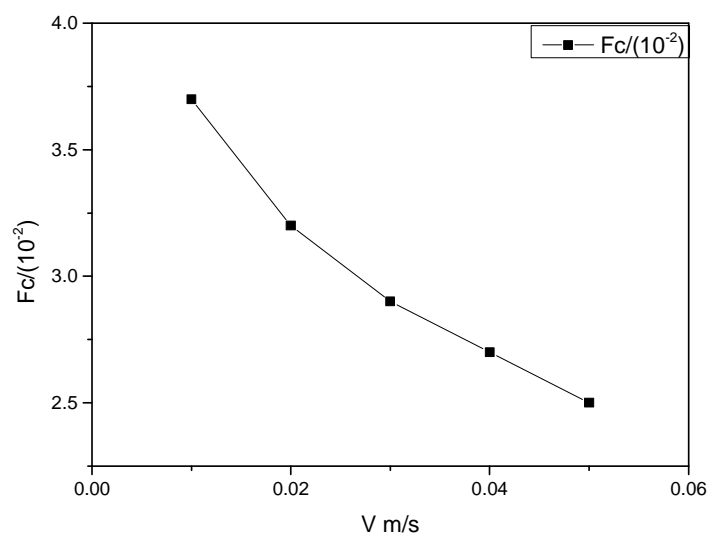

Fig.6 The average field synergy number with fluid velocity

Effect of Fluid Velocity on the Field Synergy Degree. In order to explore the effect of fluid velocity on the mass transfer performance of the hollow fiber membrane modules, the amplitude of $6 \mathrm{~mm}$ and the vibration frequency of $10 \mathrm{~Hz}$ were fixed, the inlet velocity of $0.01 \mathrm{~m} / \mathrm{s}, 0.02 \mathrm{~m} / \mathrm{s}, 0.03 \mathrm{~m} / \mathrm{s}$, $0.04 \mathrm{~m} / \mathrm{s}, 0.05 \mathrm{~m} / \mathrm{s}$ were adopted, the results were shown in Fig. 6 . With the increase of fluid velocity, the average field synergy number shows a descending tendency. The reason is that the relative change of fluid velocity caused by vibration is smaller, which leads to a reducing degree in the secondary flow process and the degree of synergy between velocity field and concentration field is lower.

It should be noted that, although the increase of fluid velocity leads to a lower degree of synergy, the degree of fluid velocity increase is greater than the degree of reduction of field synergy. That is, 
the degree of Reynolds number increase is greater than the reduction of average field synergy. According to Eq.(1), the Sh number will increase with the growth of fluid velocity, which will enhance the mass transfer performance of the hollow fiber membrane modules.

\section{Conclusions}

In this paper, the field synergy principle was used to simulate the hollow fiber membrane modules under vibration conditions. The results show that:

(1) Under the condition of vibration, the average field synergy number is obviously higher than that under the non-vibration condition. With the increase of the vibration frequency and amplitude, the average field synergy number increases. Increasing vibration frequency and amplitude does not linearly improve the mass transfer performance of hollow fiber membrane modules.

(2) The degree of field synergy is related to the vibration energy consumption, and the increase of vibration energy consumption per unit mass will enlarges the degree of synergy between the concentration field and velocity field, which will enhance the mass transfer performance of the hollow fiber membrane modules. In the range of the parameters which were studied in this paper, the increase of the vibration frequency possesses a more obvious enhancement effect than that of the amplitude.

(3) With the increase of the fluid velocity, the average field synergy number shows a descending tendency. But in general, the $S h$ number will increase with the fluid velocity, which will enhance the mass transfer performance of the hollow fiber membrane modules.

\section{Acknowledgements}

This work is financially supported by the Natural Science Foundation of Shandong Province(NO. ZR2013EEL011)and the program of Green tire \& Rubber Co Innovation Center(2015GTR0019).

\section{References}

[1] Guo Z Y. Physical mechanism of convective heat transfer and its control: Synergy of velocity field and heat flux field[J]. Chinese Science Bulletin, 2000,45 (19): 2118-2122

[2] Li Z X, Guo Z Y. Field synergy theory of convection heat transfer optimization[M].Beijing: Science Press, 2010

[3] Guo Z Y, Huang S Y. Field synergy principle and new heat transfer enhancement technology[M]. Beijing: China Electric Power Press, 2004

[4] Guo Z Y, Tao W Q, Shan R K. The field synergy (condition) principle and its applications in enhancing single-phase convective heat transfer[J]. International Journal of Heat and Mass Transfer, 2005. 48 (9): 1797-1807

[5] Tao W Q, Guo Z Y. Field synergy principle for enhancing convective heat transfer-its extension and numercial verification[J]. Heat and Mass Transfer, 2002, 45: 3849-3856

[6] Zhou J J, Tao W Q, Wang D B. Qualitative analysis and quantitative discussion on evaluation index of field synergy principle[J]. Journal of Zhengzhou University, 2006, 2: 45-47

[7] Yang H, Xuan Y M. Research progress of field synergy theory applied to heat transfer enhancement[J]. Clean and Air Conditioning Technology, 2014 (2): 15-18

[8] Wu Y Y, Liu L J. Convective heat transfer characteristics and field synergy analysis in a vibrating tube[J]. Petrochemical Equipment, 2011, 40 (6): 601-605

[9] Jiang B, Tian M C, Qiu Y, et al. PIV experimental study and field synergy analysis of the external flow field in a circular tube[J]. Journal of Chemical Engineering, 2009, 60 (8): 1900-1905

[10] Gabelman A, Hwang S T. Hollow fiber membrane contactors [J]. Membr. Sci, 1999, 159: 61-106

[11]Ding Z W, Liu L Y, Ma R Y. Study on the effect of flow maldistribution on the performance of the hollow fiber modules used in membrane distillation[J]. Membr. Sci, 2003, 215: 11-23 
[12] Tao W Q, He Y L, Wang Q W, et al. A unified Analysis on Enhancing Single Phase Convective Heat Transfer with Field Synergy Principle[J]. International Journal of Heat and Mass Transfer, 2002 (45): 4871-4879

[13] Wu L B, Li Z, Song Y Z. Field synergy principle of heat and mass transfer process[J]. Chinese Science Bulletin, 2009, 54, 14: 2045-2050

[14] Meng H B, Wang F, Yu Y F, et al. Synergetic analysis of convective heat transfer enhancement in multi rotating static mixer[J]. Journal of Harbin Engineering University, 2015 (2): 282-286

[15]Zhang S H. College Physics[M]. Third Edition: Tsinghua University Press, 2009 